\title{
CONTROL SYSTEMS FOR CURRENT OPTICAL NETWORKS BASED ON GMPLS PROTOCOL
}

\author{
Sistemas de control para redes ópticas actuales basadas \\ en el protocolo GMPLS epurations
}

\author{
CAROLINA VÁSQUEZ* \\ Recibido: 19 de diciembre de 2016. Aceptado: 29 de diciembre de 2016 \\ DOI: http://dx.doi.org/10.21017/rimci.2017.v4.n7.a26
}

\begin{abstract}
Optical transport systems have evolved in many senses with the pass of years. A first advance can be seen on the transport network, which at the beginning used TDM protocol for transport. PDH was the first used protocol, but due to its equipment complexity was replaced for SDH which gives to the optical network a better management. Later, optical networks use WDM to transport data and its improvement, DWDM is currently used providing to transport networks velocities over $100 \mathrm{Gbps}$.

On the other hand, a new control plane protocol was development, MPLS and its improvement for optical networks GMPLS, which is based on the forwarding of information separated from header IP content and the label swapping between MPLS routers, allowing a faster processing of packets or optical signals over the network, this due to the use of labels on packets which contains its own routing information.
\end{abstract}

Keywords: DWDM, MPLS-GMPLS, label, SDH, ROADM, routing, RSVP-TE.

\begin{abstract}
Resumen
Los sistemas de transporte óptico han evolucionado en muchos sentidos con el paso de los años. Un primer avance se puede ver en la red de transporte, que al principio utilizó el protocolo TDM para el transporte. PDH fue el primer protocolo utilizado, pero debido a su complejidad de equipos se sustituyó por SDH que da a la red óptica una mejor gestión. Posteriormente, las redes ópticas utilizan WDM para transportar datos y su mejora, DWDM se utiliza actualmente para proporcionar velocidades de redes superiores a 100Gbps.

Por otro lado, se desarrolló un nuevo protocolo de plano de control, MPLS y su mejora para redes ópticas GMPLS, que se basa en el reenvío de información separada del contenido IP de cabecera y el intercambio de etiquetas entre routers MPLS, permitiendo un procesamiento más rápido de paquetes o señales ópticas a través de la red, esto debido al uso de etiquetas en paquetes que contiene su propia información de enrutamiento.
\end{abstract}

Palabras clave: DWDM, MPLS-GMPLS, etiqueta, SDH, ROADM, enrutamiento, RSVP-TE.

\section{INTRODUCCIÓN}

$\mathrm{T}_{1}$ HE INCREASED volume of data traffic generated on last twenty years pushed the evolution of optical transport systems to support high data traffic and transmission capacity. That is why optical systems have evolved through the years, passing for Time Division Multiplexing systems for digital transmissions, which at first were based on the Plesiochronous Digital Hierarchy, but due to the difficulties to identify a low level traffic line on a high order traffic, (because of the necessity to use a high number of demultiplexers) and the lack of standardization, led to leave behind the use of $\mathrm{PDH}$, to begin to use the Synchronous Digital Hierarchy standard as a better solution for transport networks, which provides an efficient management of the network. To reduce electronical complexity of SDH for transmissions over 10Gbps, service providers (SPs) begin to use

Ingeniera de telecomunicaciones de la Universidad de Antioquia, con énfasis en la línea de comunicaciones ópticas y conocimiento en el diseño de redes de transporte DWDM y redes GPON. Correo electrónico: carolina.vasquezc@udea.edu.co 
Wavelength Division Multiplexing, which is a great solution for high capability networks. Sometime later, to improve the transmission capacity, WDM evolved to DWDM multiplexing which today is allowing transmission velocities over $400 \mathrm{Gbps}$.

Having a solution for transport networks of high capacities on the transport data plane, researchers focus on the control data plane to reach a faster processing routing over the network. This introduced the MPLS and GMPLS protocols definition, where the last one protocol is an improvement of the first one for implementations of optical networks. This new protocol was created around 2000 by the Internet Engineering Task Force (IETF) and operates between link layer and transport layer of the OSI model, allowing a faster routing over the network.

Over the last years, IP routing has evolved to include new functionalities under MPLS, extending it as a control plane that can be used not just with routers, but also with legacy devices such as $\mathrm{SDH}$, OXCs and OADMs equipment, this offer the necessary standardized common control plane which simplifies operation and management, and provides several deployment scenarios form overlay to peer models (or a combination of them). An Overlay model hides inner network details, in that sense uses two separated control planes, one of them operating in the core of the network and the other one operating between the core and edge devices. On the other hand a Peer model allows to edge devices participate on routing decisions, this means that there is just one control plane [1].

This paper focus on the explanation of functionalities of MPLS, its evolution to GMPLS and is outlined as follows. In Section II, a state of the art of optical transport systems is presented. Section III presents an overview of MPLS and GMPLS, while Sections IV and V present an explanation of ROADM operation and conclusions, respectively.

\section{STATE-OF-THE-ART}

The following state-of-the-art shows the evolution of optical transport systems beginning at time division multiplexing, its characteristics and protection schemes, and later wavelength division multiplexing, its components and configurations.

\section{A. Time Division Multiplexing (TDM)}

Time division multiplexing is a method of putting multiple data streams on a single signal by separating the signal into many segments, each having a very short duration (time slot). Each individual data stream is reassembled at the receiving end based on the timing. This way to operate allows multiplexing several low velocity channels into a very fast channel, to be transmitted through the network [2].

TDM is used to transmit digital signals where the basis unit is $64 \mathrm{kbps}$ (DS0) which is equivalent to one voice channel. TDM for a voice channel consists on assign a time slot to each channel for transmission which repeats every $125 \mu \mathrm{s}$, the multiplexed signal forms a frame, and each frame consists of a given number of time slots. With the purpose of bring together the biggest quantity of channels using multiplexing, can be explained two different multiplexing hierarchies.

\section{1) Plesiochronous Digital Hierarchy (PDH)}

T1-Carrier: This is an American standard where 24 telephone channels or any type having an output rate of $64 \mathrm{kbps}$ are combined. The frame is organized with 24 channels of 8 -bit, one bit is added for frame alignment, giving a total of 193 bit/frame. The output rate is $1544 \mathrm{kbps}$ and the signaling is done by stealing the least significant bit of every sixth frame each channel, which gives a signaling rate of $8000 / 6=1333.3 \mathrm{~Hz}$ [3].

E1-Carrier: This is a European standard where 30 telephone channels of $64 \mathrm{kbps}$ are multiplexed,
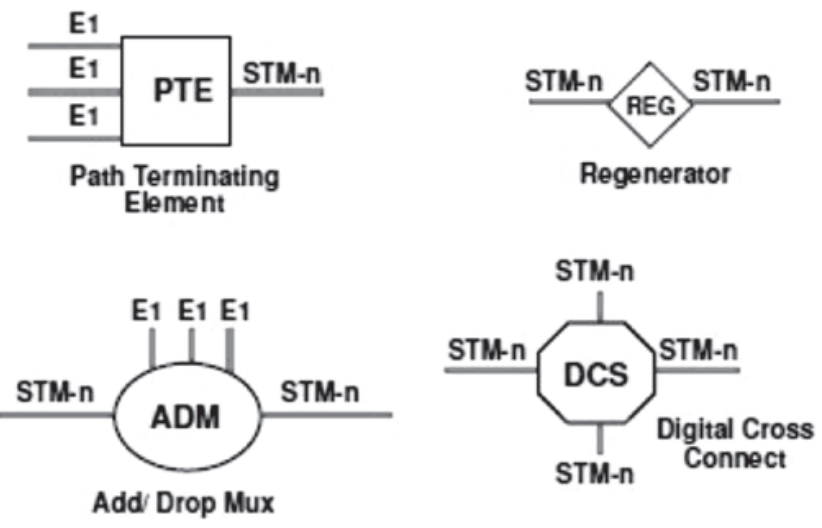

Fig. 1. SDH network elements.

Rev. Ingeniería, Matemáticas y Ciencias de la Información Vol. 4 / Núm. 7 / enero-junio de 2017; pág. 87-98 
and two additional channels for timing and signaling are used. The output rate is $2048 \mathrm{kbps}$ and channel 1 and channel 16 are used for frame alignment, signaling and monitoring.

\section{2) Synchronous Digital Hierarchy (SDH)}

Since in PDH access to individual channels from signals of superior order requires a demultiplexation and subsequent full multiplexing, more expensive equipment is needed to locate where it is desired remove and insert channels, $\mathrm{SDH}$ becomes a great solution for this problem, this is a standard that allows the integration of different TDM signals allowing its transmission over optical fiber.

SDH supports other hierarchies as PDH, ATM, FDDI, among others, which makes it a very flexible hierarchy. SDH also defines the transmission rate, signal format, multiplexing structure, line coding, optical parameters and performance standards of equipment and network management [4].

Fig. 1 shows the basic components of a SDH network. Path Terminating Element (PTE) is the endpoint device where lower speed channels entering and leaving the SDH network, the Add/ Drop Mux has the ability to download and insert low speed channels, and the Digital Cross Connect may connect individual frames E1.

With knowledge of the components of a SDH network, it is now possible to identify all SDH network sections, considering the use of the above explained components.

Fig. 2 shows three important sections on a SDH network: Path Section is created and terminated by a PTE at both ends of the link, the Multiplex Section starts where the SDH frame is assembled and ends where the frame is disassembled (PTE or ADM), and the Regenerator Section is located between a PTE or ADM and a Regenerator.

Modern SDH networks include several automated mechanisms for protection and recovery failure systems. A problem on a link or a network element does not cause the collapse of the entire network, which could be an economical disaster for the provider. These protection circuits are also controlled by a management system. Following are some protections schemes for lineal SDH networks:

Path switching: This protection uses two links and duplicates the signal on the working and protection paths. Allows to protect low capacity services individually.

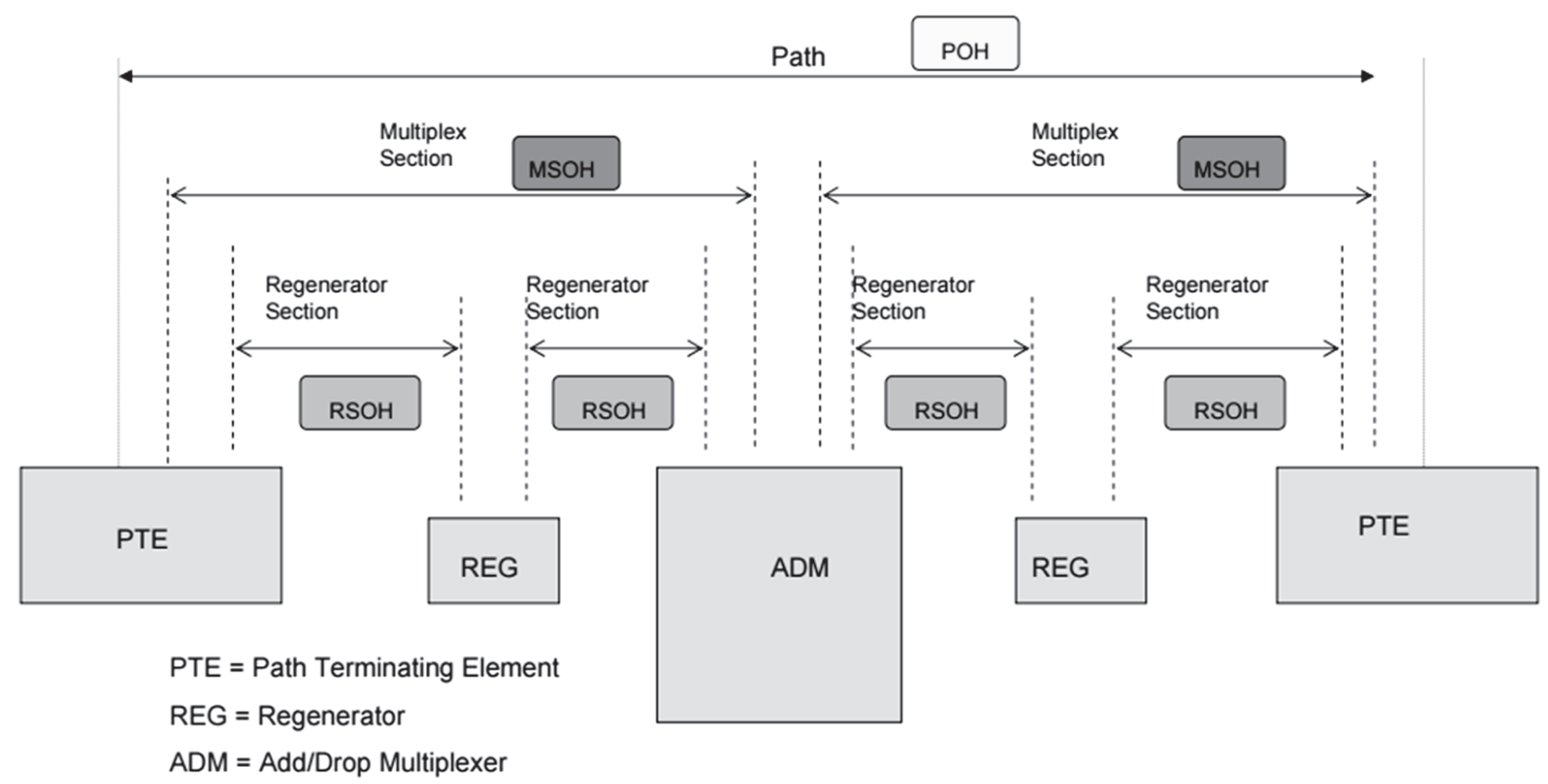

Fig. 2. SDH network sections. 
1+1 protection switching: It requires twice as many resources in the system, providing dedicated protection. Traffic is initially sent by both the working path as the protection path. If a loss of traffic is detected on the receiving end, switching process begins towards the protection path. The protection path cannot be used to transmit any traffic.

Fig. 3 shows the different protection schemes mentioned below. Fig. 3(a) details the path protection switching, where the dashed line represents the use of the protection path in case of failure. Fig. 3(b) represents the $1+1$ protection switching.

1:1 protection switching: It is a double ended scheme. Traffic is initially sent by the working path only. A fault is detected on the opposite end when it do not receive traffic for an extended period of time. A signal is sent to the transmitting end that triggers protection switching, sending traffic to the protection path at both ends. This means that lowpriority traffic can be carried by the protection link while travel by the operating channel. This traffic will be lost when a protection switching process starts.

In this sense, also N:M protection switching can be done, this consists on $\mathrm{N}$ working paths and $\mathrm{M}$ protection paths, where the operational principle is the same for 1:1 protection switching.

Functionality of 1:1 and N:M protection schemes are detailed on Fig. 4(a) and 4(b), respectively.

For ring topology the following are the most used protection switching schemes:

Multiplex Section-Shared Protection (MS-SP) ring: Employs just the half capacity at each sense. When there is a fault, the adjacent nodes detected it and send the traffic on the other direction. This procedure is shown on Fig. 5(a), where line green represents the traffic re-routed.

Subnetwork Connection Protection (SNCP) ring: Traffic is sent over the working path and the protection path $(1+1$ protection), protecting the system against fiber cut or node failures [5].

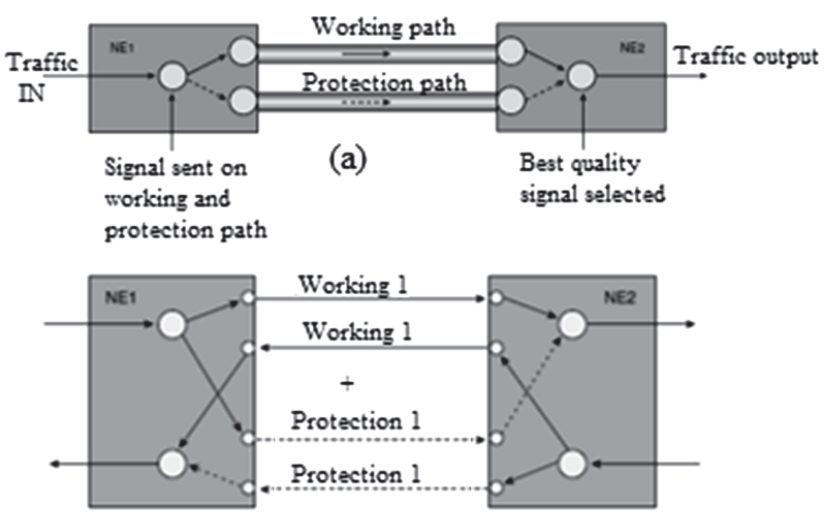

(b)

Fig. 3. Path switching [a] and 1+1 protection switching [b].

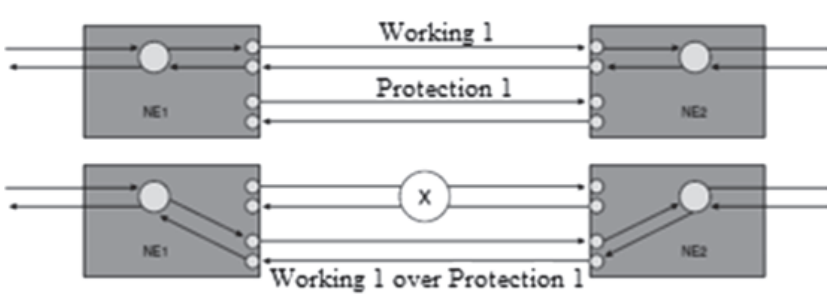

(a)

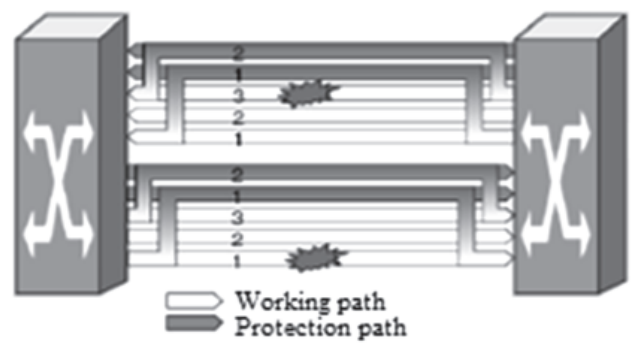

(b)

Fig. 4. 1:1 protection [a] and N:M protection [b]

Since demand for high-speed connections is growing up due the increasing number of people using data services and devices connected to Internet (IOT), TDM systems cannot supply the demand of a higher bit rate over 10Gbps, because of the electronical devices complexity. In that sense, WDM rises as a better and economical way to transmit information at a bigger bit rate than 10Gbps over backbone networks.

\section{B. Wavelength Division Multiplexing (WDM)}

Wavelength division multiplexing is a technology of numerous optical carrier signals of varying

Rev. Ingeniería, Matemáticas y Ciencias de la Información Vol. 4 / Núm. 7 / enero-junio de 2017; pág. 87-98 

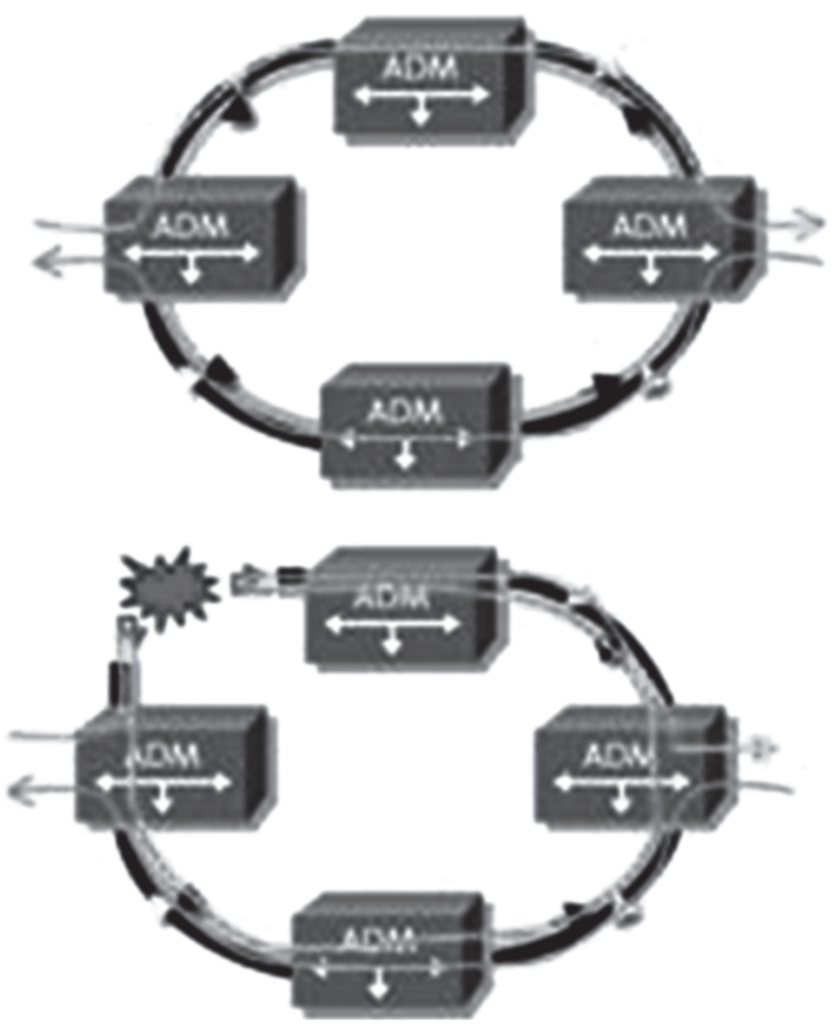

Fig. 5. MS-SP ring (a) and SNCP ring [b].

wavelengths (colors) of laser light, onto a single optical fiber. WDM enables bidirectional communication as well as multiplication of signal capacity in enterprise, access, backhaul, metro and longhaul networks by creating virtual fibers.

WDM is now recognized as the layer 1 transport technology in all tiers of the network. It offers lowcost transport for all applications and services, scales easily in terms of capacity and reach and provides rapid protection against any fiber plant failure [6].

Currently, there are two types of WDM: Coarse WDM (CWDM) and Dense WDM (DWDM).

CWDM: operates with 18 channels which have a channel spacing of $20 \mathrm{~nm}(2500 \mathrm{GHz})$. The first channel is located at $1270 \mathrm{~nm}$ and the last one is at $1610 \mathrm{~nm}$. Each channel has a capacity of $2.5 \mathrm{Gbps}$ for a distance of $50 \mathrm{~km}$ [7].

DWDM: operates with 40 channels at $0.8 \mathrm{~nm}(100$ $\mathrm{GHz}$ ) channel spacing or 80 channels at $0.4 \mathrm{~nm}$ (50 $\mathrm{GHz}$ ) spacing. Each channel can have a capacity of $2.5,10,40$ or $100 \mathrm{Gbps}$. Today's DWDM systems uses $16,32,64,128$ or more wavelengths in the $1550 \mathrm{~nm}$ window [8].

The essential components of a WDM system are primarily those of any network, like transmitters, optical fiber (for optical communications) and receivers. In case of WDM technology, the transmitters are laser sources with stable tunable wavelengths. Before sending the signal through optical fiber and multiplexers mix the wavelengths, at the receiver end there are photo detectors and wavelength demultiplexers. In addition, the system would require other components such as switches, modulators, optical amplifiers, isolators, etc. Fig. 6 shows a real configuration for a WDM link, where the optical signals generated on transmission end passes combines on the multiplexer and are send through the optical fiber to the receiver end, but in that travel signal is amplified several times depending of the distance between transmission and reception ends.

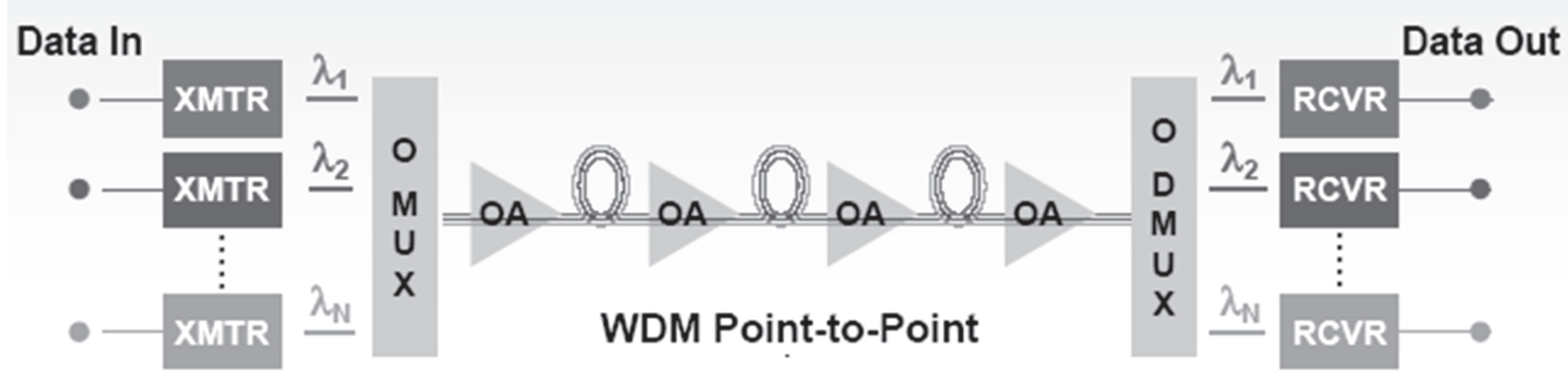

Fig. 6. Basic topology of WDM networks 


\section{Multi-Protocol LABEL SWITCHING}

At the end of nineteenth century data networks had four layers: IP for carrying applications and services, asynchronous transfer mode (ATM) for traffic engineering, SDH for transport, and dense wavelength division multiplexing (DWDM) for capacity (Fig. 7). To remove ATM and SDH layers on high capacity transport networks is necessary that this layers bypass their functionalities to routers, DWDM and optical cross-connected (OXC), where OCXs are the most common option for switching multigigabit or even terabit data streams. This new conformation of data networks is more cost-efficient and allows to transport a wide range of data streams and high capacity traffic.

Nowadays the predominant traffic carried is IP based, because of this the development of fast router technologies is very important to supply the demand of high velocities connections for today costumers. In that sense, multiprotocol label switching (MPLS) is today implemented over high capacity and high traffic volume networks, giving them new functionalities at level of plane control which simplifies operations and management, and reduces the cost of operations.

MPLS is based on the forwarding of information separated from header IP content and the label swapping between MPLS routers. Label switch routers (LSRs) forward data using the label carried by the data. This label and the port on which the data was received for the LSR, is used to determine the output port and outgoing label for the data to move over the MPLS network. Each label defines a packets flow between two end points where each flow is different and is called forwarding equivalence class (FEC). Each FEC has a specific route through the LSRs on the network, because of this, MPLS is considered a connection oriented protocol where FEC contains the QoS specifications and requirements of packets flow which allows to determinate the quantity of resources to reserve on the network [9].

The MPLS framework includes applications such as constraint based routing. This is a mix of different extensions to IP link-state routing protocols, specially Open Shortest Path First (OSPF) and Intermediate System to Intermediate System (IS-IS), with Reservation Protocol (RSVP) or Constraint-based Routing Label Distribution Protocol (CR-LDP) signaling as the MPLS control plane and the Constrained Shortest Path First (CSPF) heuristic.

Before to forward information over the network is necessary to stablish a label switching path (LSP) between routers that transmits the FEC. These LSPs are like transport tunnels on the MPLS network.

The extensions to OSPF and IS-IS allows nodes to exchange information about network topology, resource availability and routing tables. This information is used by the CSPF heuristic to compute paths depending to specified resource and/ or policy constraints. For example, for the creation of the LSP are needed either RSVP-TE or CR-LDP which is used to establish the label forwarding state along the routes computed by a CSPF-based algorithm, and later the MPLS data plane forwards the information along the established LSP.

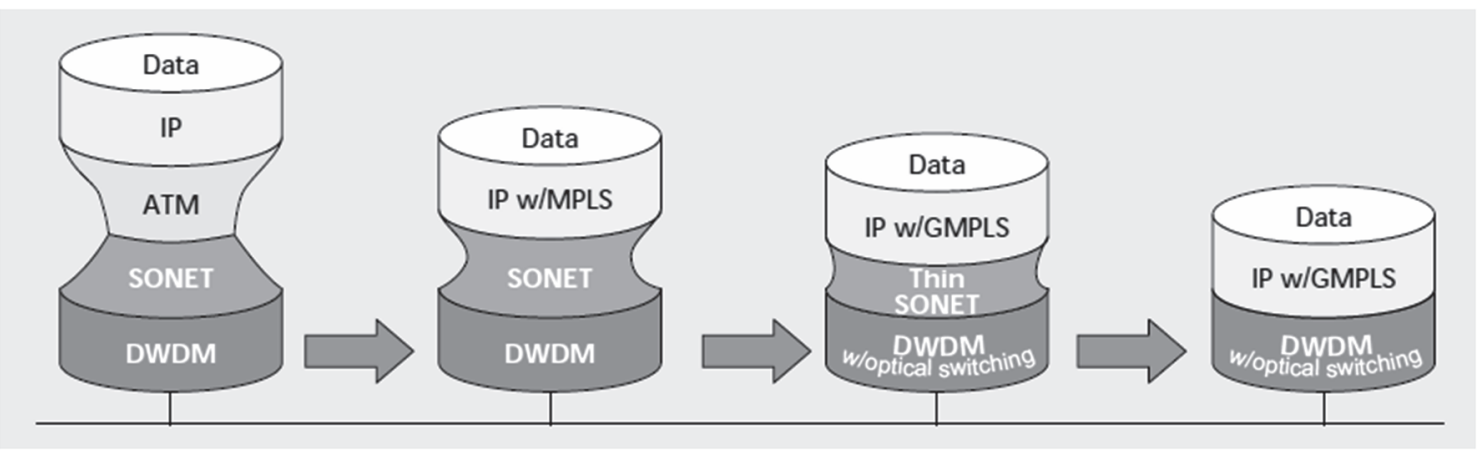

Fig. 7. Evolution of data networks [10]. 
As mentioned above ATM layer is removed as the layer in charge of traffic engineering, and constraint-based routing provided by MPLS to IP routing replaces this functionality where at the same time gives to the network a fast reroute, offering an alternative to SDH as a scheme for protection.

Label Edge Routers (LERs) provide the connection of each IP client, this routers analyze and classifies the input IP packets just considering IP destination address and QoS requirements and later puts the MPLS label at each IP packet, to identify the LSP where the packet is. This is, the LER decides the entire LSP which packets will take over the MPLS network. After assigning the label, LER passes the packet to the LSR which is located on the core of the MPLS network (Fig. 8).

Following are some benefits of MPLS networks:

1) Traffic engineering for ISPs to moving data traffic from the shortest path (calculated by routing protocols) to less congested paths or less susceptible to failures paths, i.e., that refers to the process of selecting which paths will takes the data, seeking the balance of traffic on all links, routers and switches, avoiding the overloaded of them.

2) Transport of differenced traffic with several classes of service (CoS), which defines the requirements of links to provide an adequate quality of service (QoS).
3) Support of virtual personal networks (VPN). MPLS offers a simple mechanism to create VPN, since provides the creation of tunnels or virtual circuits along an IP network.

Once seen the MPLS concept, we can talk about generalized MPLS (GMPLS) networks. This protocol not just supports packets switching devices but also devices that performs switching in the time, wavelength and space domains, i.e., GMPLS seeks total integration of control plane of IP switching networks and optical networks such as SDH and DWDM, resulting in intelligent next generation optical networks, whose final outcome will be the integration of IP directly on DWDM (Fig. 7).

Deploying GMPLS on an optical network is a combination of LSRs and optical switches, and LSPs and optical trails, where wavelengths (lambdas) assume the role of labels and optical switches toggle wavelengths from an input to an output port. For the establishment of an optical trail is necessary to configure all optical switches on the GMPLS networks to map one input lambda and port to an output lambda and port. As LSRs, optical switches need routing protocols like OSFP or IS-IS to exchange information about network topology (link-state topology) to compute paths. Optical switches also need signaling protocols like RSVP and LDP to automate the path establishment process.
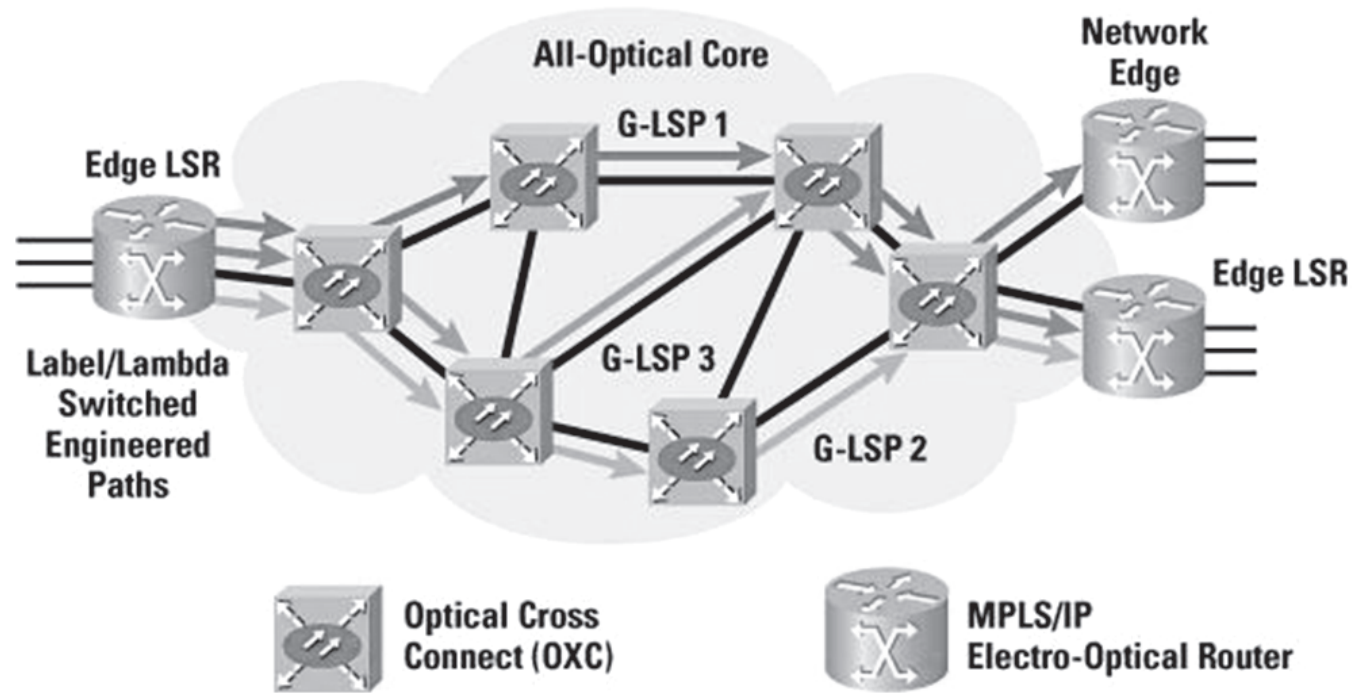

Connect (OXC)

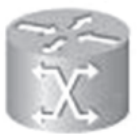

MPLS/IP Electro-Optical Router

Fig 8. MPLS network example [11]. 
In that sense MPLS networks need some modifications and additions on the routing and signaling protocols to achieve an adequate match with optical switches for the development of GMPLS networks. Following are some of this modifications:

1) Deployment of a new Link Management Protocol (LMP) whose purpose is to solve issues related to link management in optical networks which use optical switches. LMP provides four basic functions for a node pair: control channel management, this is used to establish and maintain connectivity between adjacent nodes on the network. This functionality allows to send the component links of a bundled link separated of the control channel, i.e., the component links and associated control channel need not be transmitted over the same physical medium. Other functionality of LMP is the link verification which is used to verify the physical connectivity of the component links. Link property correlation of function link properties such as link IDs, protection schemes and priorities between adjacent nodes is provided by means of the Link Summary message of LMP. Finally LMP provides the functionality to isolate link and channel failures independent of the data format.

2) Design an enhanced OSPF/IS-IS routing protocols to show the availability of optical resources in the networks, such as, bandwidth on wavelengths, link protection schemes and fiber IDs.

To help understand the routing enhancements needed, a brief description of link state protocols such as IS-IS and OSPF will be given. A link state protocol allows all nodes on a network (MPLS switches, OXCs, etc.) to know not just the information about network topology but also about availability of resources such as the number of total wavelengths and the number of unused wavelengths for each link. With this information each node can build a picture of the network referred as the link state database. Once all nodes on the network knows the link state database, each node uses this information to build its own forwarding table. These tables allow nodes to know how to forward an incoming packet. Database will be resynchronized when a link is added or removed of the topology, and nodes will recalculate their forwarding tables using the updated information in the link state database. In that sense, OSPF extension is used for the realization of multilayer traffic engineering.

3) Design an enhanced RSVP or CR-LDP signaling protocol for traffic engineering purpose, to allow an explicit and specified LDP over the optical network. RSVP is a protocol for connection establishment and network resource reservation for IP networks, where the resources reservation depends of the QoS needed by the service that will be transported over the network. RSVP can be used on MPLS and GMPLS networks by using traffic engineering extensions [10].

The main control messages in a RSVP are Path and Resv messages, originated from the senders and receivers, respectively. Path messages follow the route computed by the routing protocol and carry RSVP data to provide receivers with the description of the sender and traffic flow. Upon receipt of a valid Path message, each intermediate RSVP node updates their path state entry for the sender before forwarding the appropriately updated path message towards the receiver. After receiving a Path message, the receiver can make a reservation by sending a Resv message back to the source.

For MPLS networks, RSVP protocol adds new messages such as Label Request carried in Path messages and Label in Resv messages. Additional new data messages were defined to support explicit routing and to record the route taken by particular messages. These extensions to RSVP essentially made the protocol more adept at supporting traffic engineering (TE) and this version of RSVP is commonly referred to as RSVP-TE. This new version defines a hello extension, which are messages exchanged at regular intervals of time by adjacent RSVP nodes. This messages can help to know when a RSVP node could not have communication with its neighbors. When a node does not detects a Hello message within a certain interval it is said that the node could not have communication with its neighbors [12].

Following are some RSVP enhancements needed to support TE on GMLPS networks: suggested label, notify messages and bidirectional LSP setup.

Rev. Ingeniería, Matemáticas y Ciencias de la Información Vol. 4 / Núm. 7 / enero-junio de 2017; pág. 87-98 
The suggested label refers to a label suggested by an upstream node in the GMPLS network. This is very useful when it is desired to set up a bidirectional LSP using transmitter and receiver interfaces which sharing the same physical port (e.g., WDM transponders Tx/Rx pair) or to set up an LSP transiting certain kinds of optical switching equipment, where there is some latency associated with configuring the switching fabric. The suggested label concept allows an upstream node along a service path to start configuring its hardware with the suggested label before the downstream node communicates a label to it. Early configuration offered by a suggested label can reduce setup latency, and may be important for restoration purposes as well, where alternate LSPs may need to be rapidly established.

The notify messages provides to GMPLS networks a mechanism for informing non-adjacent nodes about LSP failures, giving to the network a faster reaction to possible failures which at the same time provides major reliability. This can be done by intermediate nodes (nodes where neither originates nor ends a connection) which only passing information about the failure to the node responsible for restoring the connections, without processing the message which could delay notifications and restoration of the service.

The bidirectional LSP setup is a new functionality of GMPLS networks, this because on traditional MPLS, LPSs are unidirectional. So to establish a bidirectional LSP can be possible using two unidirectional LSPs in opposite directions [13].

4) Deployment of some scalability enhancements such as hierarchical LSP formation, link bundling, and unnumbered links [14].

\section{RECONFIGURABLE OPTICAL ADD/DROP MULTIPLEXER}

To add flexibility, DWDM networks evolved into multi-node linear and ring configurations. When a wavelength reached a node, it could be stopped at the node or pass through the node. In addition, wavelengths could be added into the DWDM stream at intermediate nodes. This introduces the concept of wavelength add/drop in nodes which well-known as optical add/drop multiplexers or OADMs [15].

Rev. Ingeniería, Matemáticas y Ciencias de la Información

Vol. 4 / Núm. 7 / enero-junio de 2017; pág. 87-98
An optical add-drop multiplexer (OADM) is a device that adds one or more wavelengths to the DWDM signal or drops one or more wavelengths passing that signals to another path without the ability of pre-configures the wavelengths to be added, dropped or passed through the fiber.

There are three stages in a traditional OADM: an optical demultiplexer, an optical multiplexer, and between them a method of reconfiguring the paths between the optical multiplexer and a set of ports for adding and dropping signals.

In that sense, a Reconfigurable OADM (ROADM) allows a dynamic configuration for extraction or insertion of wavelengths to the DWDM stream, this using a Wavelength Selective Switch (WSS). The main function of a WSS is independently switch each of the $M$ wavelengths between a common port and one of the $\mathrm{N}$ ports of switching, controlling the attenuation for each one. Most of WSS works with the International Telecommunications Union (ITU) grid of 100 or $50 \mathrm{GHz}$ on $\mathrm{C}$ or L band. The WSS provides quick service start-up, remote cross-connect and DWDM mesh networking [16].

The ROADM scheme also allows inputting or outputting a single wavelength or wavelengths group via the fixed port. ROADM can configure as required without affecting traffic. It's also used for remote configuration or reconfiguration via Network Management System (NMS). This type of OADM is very flexible in rerouting optical streams, bypassing faulty connections, allowing minimal service disruption and the ability to adapt or upgrade the optical network to different WDM technologies.

Figure 9 describes the operational principle of one particular node on a ring network using ROADMs. For example, where a DWDM signal comes from left to right, the signal goes to the input port of the WSS and right there two process can be done, the first one is pass the signal from the input to the output port, and for the second process some wavelengths of the DWDM signal can be added o dropped. The same process is done in the sense right-left.

The WSS can select any color to be routed towards the drop side of the node or bypass to the next node. The input port can receive the whole 


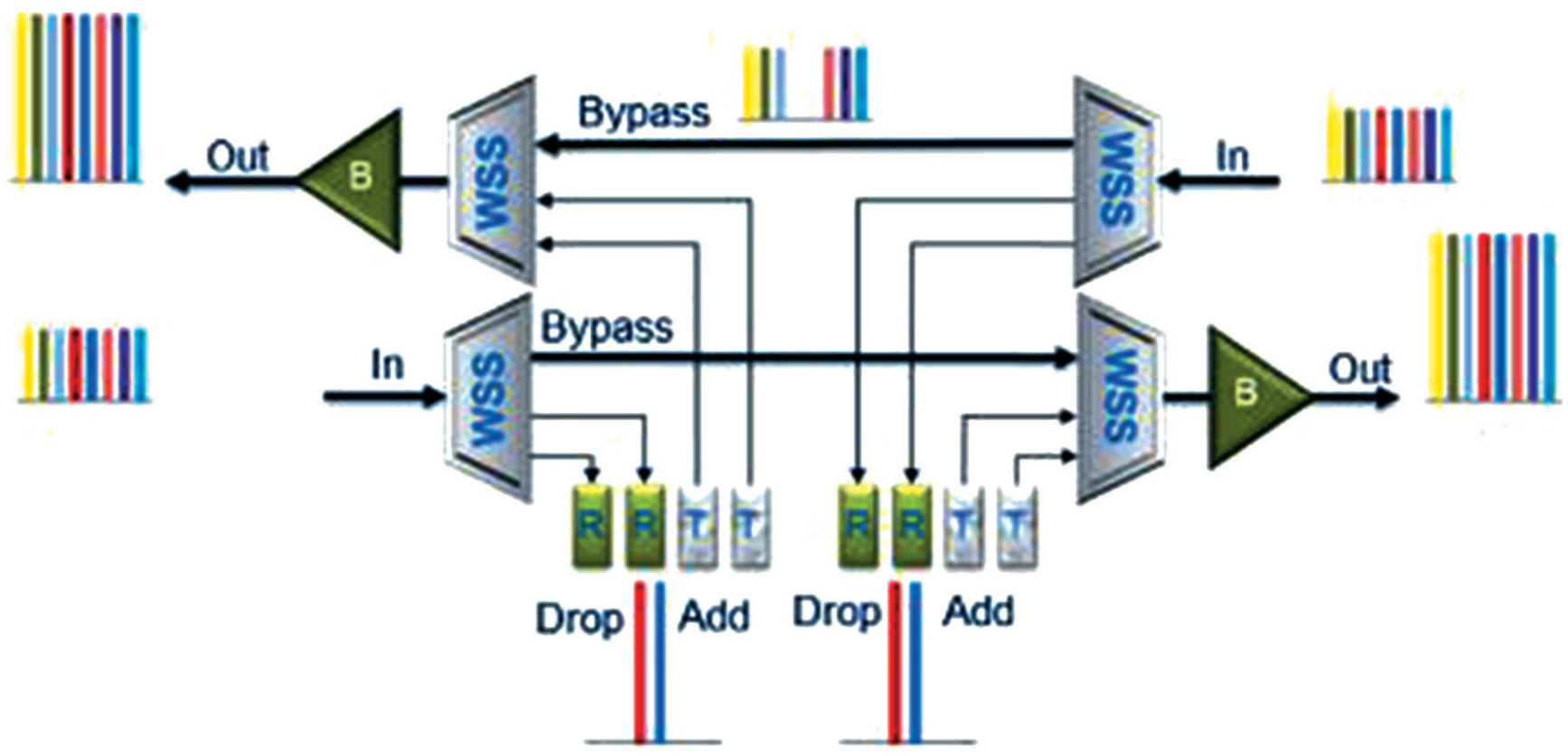

Fig. 9. ROADM basic operation.

C band and the drop (or add) ports can drop (or add), bypass or block any channel as chosen.

The ROADMs can have different functionalities, giving them flexibility and major grade of quality. The following are some of this functionalities:

Colorless: There are several variations for building colorless ROADMs, but they typically involve the use of additional WSSs in place of multiplexers and demultiplexers, the result of this change is that any wavelength (color) can be assigned to any port at the add/drop site, completely by software control and without a technician on site.

Directionless: Directionless ROADMs allows any wavelength to be routed to any direction served by the add/drop node, by software control, and without physical rewiring.

Contentionless: When two wavelengths of the same color converge at the same WSS structure at the same time can be a wavelength blocking and this causes network contention. Operators must avoid this potential blocking/contention situation by partitioning the add/drop structures so that different colored wavelengths are associated with different structures, thus eliminating the possibility for two wavelengths equal to converge on the same add/drop multiplexer. This can resolve wavelength contention but it means that operators sacrifice a level of flexibility and may require additional add/drop multiplexers to accommodate particular wavelength channels. A contentionless architecture, by contrast, allows multiple copies of the same wavelength on a single add/drop multiplexer.

Flexible grid: a conventional ROADM uses a grid of 100 or $50 \mathrm{GHz}$ (in some cases $25 \mathrm{GHz}$ or even $12.5 \mathrm{GHz}$ ), but a flexible grid or gridless ROADM involves a more granular use of the spectrum to support velocities over $100 \mathrm{Gbps}$. In case of $40 \mathrm{Gbps}$ the channel spacing will be of $75 \mathrm{GHz}$ and $1 \mathrm{Tbps}$ will require just 150GHz channel spacing [17].

A ROADM with colorless, directionless, contentionless and flexible grid functionalities is so-called CDC gridless ROADM (colorless, directionless, contentionless - any wavelength, any node, any time) and is fully flexible providing nonblocking. CDC gridless ROADMs (Fig. 10) are the foundation of a fully automated optical network, and they allow for intelligent interworking with the MPLS technology that rides on top [17].

Table 1 can show the evolution of ROADMs from their first generation to current ROADMs with their different characteristics and functionalities for each generation. 
Table 1. Roadm generations [17]

\begin{tabular}{|c|c|}
\hline ROADM generations & Characteristics \\
\hline 1st generation: Wavelengths Blocker based ROADMs & $\begin{array}{l}\text { - } 2 \text { degree nodes only } \\
\text { - } 100 \mathrm{GHz} \text { channel spacing } \\
\text { - Add/Drop only } \\
\text { - No channel equalization capability } \\
\text { - Neither colorless nor directionless }\end{array}$ \\
\hline 2nd generation: Planar Light-wave Circuit (PLC) based ROADMs & $\begin{array}{l}\text { - } 2 \text { degree nodes and very limited multi degree } \\
\text { functionality } \\
\text { - } 100 \mathrm{GHz} \text { channel spacing } \\
\text { - } \text { Add/Drop only } \\
\text { - Channel equalization capability } \\
\text { - Neither colorless nor directionless node support }\end{array}$ \\
\hline 3rd generation: WSS 1:N based ROADMs & $\begin{array}{l}\text { - Multi degree node support } \\
\text { - } 50 \mathrm{GHz} \text { and } 100 \mathrm{GHz} \text { channel spacing } \\
\text { - Channel equalization capability } \\
\text { - Colorless and directionless node support }\end{array}$ \\
\hline $\begin{array}{l}\text { 3rd+ generation: WSS Liquid Crystal on Silicon (LCoS) } \\
\text { based ROADMs }\end{array}$ & $\begin{array}{l}\text { - Multi degree node support } \\
\text { - Flexible channel spacing } \\
\text { - Future proof on } \\
\text { - Channel equalization capability } \\
\text { - Colorless and directionless node support } \\
\text { - Contentionless node support }\end{array}$ \\
\hline
\end{tabular}

\section{Conclusion}

The use of GMPLS on current data networks, works such a bridge between the IP and optical layers, allowing a parallel growth of services over the IP and optical domains. GMPLS provides a faster and cheaper way to operate over IP networks, giving to the network a better operational efficiency, as well as increasing economic opportunities for services providers, due to the faster processing of packets over the GMPLS network. The use of ROADM nodes on GMPLS networks simplify even more the network operation, because it allows the implementation of control points distributed in the DWDM transport network, specifically the use of CDC gridless ROADMs improves the GMPLS control plane, providing it more flexibility.

\section{REFERENCES}

[1] International Union of Telecommunications, «Spectral grids for WDM applications: DWDM wavelength grid,» 2012.
[2] M. Rouse, «time-division multiplexing,» 2005. [Online]. Available: http:// whatis.techtarget. com/definition/time-division-multiplexing-TDM.

[3] N. Massa, «Fiber Optic Telecommunication,» in fundamentals of photonics, Springfield: University of Connecticut, 2000, pp. 293-347.

[4] FiberOptic.com, «SONET/SDH.» [Online]. Available: http://www.fiberoptic.com/adt_sonet_sdh.htm.

[5] J. M. Domínguez, «Synchronous digital hierarchy (SDH),» 2004. [Online]. Available: http:/ / www. mailxmail.com/curso-jerarquia-digital-sincronasdh/esquemas-proteccion-2.

[6] ADVA Optical Networking, «WDM.» [Online]. Available: http://www.advaoptical.com/en/ products/technology/wdm.aspx.

[6] International Union of Telecommunications, «Spectral grids for WDM applications: CWDM wavelength grid,» 2003.

[7] G. Papadimitriou, C. Papazoglou, and A. Pomportsis, "Optical Switching: Switch Fabrics, Techniques, and Architectures,» J. Ligthwave Technol., vol. 21, no. 2, pp. 384-405, 2003.

[8] Z. Zhou, K. Chen, and L. Zheng, «GMPLS RSVPTE Signaling Recovery with Graceful Restart in 
Optical User Network Interface,» in 2007, Optical Fiber Communication and the National Fiber Optic Engineers Conference.

[9] 0. Komolafe and J. Sventek, «Overview of Enhancements to RSVP-TE to Increase Control Plane Resilience,» in 2007, GMPLS Performance: Control Plane Resilience, Workshop on, pp. 1-6.

[10] A. Banerjee et al., «Generalized Multiprotocol Label Switching: An Overview of Signaling Enhancements and Recovery Techniques,» IEEE Commun. Mag., vol. 39, no. 7, July. 2001.

[11] F. Palmieri, «GMPLS Control Plane Services in the Next-Generation Optical Internet,» Internet Protoc. J., vol. 11, no. 3, 2008.

[12] A. Banerjee et al., «Generalized Multiprotocol Label Switching: An Overview of Routing and Management Enhancements,» IEEE Commun. Mag., vol. 39, no. 1, Jan. 2001.
[13] S. Gringeri and B. Basch, «Flexible Architectures for Optical Transport Nodes and Networks,» IEEE Commun. Mag., vol. 48, no. 7, July 2010.

[14] Fiber Optic Solution, «Tutorial of OADM,» 2016. [Online]. Available: http://www.fiber-opticsolutions.com/tag/roadm.

[15] S. Perrin, «The Need for Next-Generation ROADM Networks,» 2010.

[16] ADVA Optical Networking, «ROADM.» [Online]. Available: http://www.advaoptical. com/en/ products/technology/roadm.aspx.

[17] J. Fowler, «Next Gen optical networks,» 2012. 